\title{
Estudio del polimorfismo MTHFR C677T en recién nacidos con cardiopatías congénitas aisladas, en una población colombiana
}

\author{
Reggie García-Robles ${ }^{1}$, Paola Andrea Ayala-Ramírez ${ }^{1}$, Victoria Eugenia Villegas ${ }^{2}$, \\ Marleny Salazar ${ }^{3}$, Jaime Bernal ${ }^{1}$, Federico Núñez ${ }^{4}$, Víctor Caicedo ${ }^{4}$, Sonia Pachón ${ }^{4}$ \\ SANDRA RAmíreZ², Martha BermúdeZ ${ }^{1}$
}

\section{Resumen}

Introducción: El estudio del papel de polimorfismos en genes de las vías metabólicas de la homocisteína-metionina y el ácido fólico en anomalías congénitas, es cada vez más importante debido a que sus efectos podrían ser modulados.

Objetivo: Determinar si la presencia del polimorfismo C677T en el gen de la metilentetrahidrofolato reductasa (MTHFR) se asocia con el desarrollo de cardiopatías congénitas aisladas.

Métodos: Se compararon las frecuencias alélicas y genotípicas del polimorfismo en 34 recién nacidos con cardiopatías congénitas aisladas y en 102 individuos sanos. La genotipificación se hizo mediante la reacción en cadena de la polimerasa (PCR) y se determinó el genotipo por medio de la técnica de polimorfismo de longitud de los fragmentos de restricción (RFLP).

Resultados: No se encontraron diferencias estadísticamente significativas en las frecuencias alélicas ni genotípicas entre los grupos de casos y controles. Sin embargo, se observó una tendencia estadística para un posible efecto protector del genotipo TT.

Instituto de Genética Humana, Facultad de Medicina, Pontificia Universidad Javeriana, Bogotá, D.C., Colombia.

2 Universidad Colegio Mayor de Nuestra Señora del Rosario, Bogotá, D.C., Colombia.

3 Programa de Licenciatura en Biología y Educación Ambiental, Universidad del Quindío, Armenia, Colombia.

4 Cirujano cardiovascular, Fundación Clínica Shaio, Bogotá, D.C., Colombia. 
Palabras clave: cardiopatía congénita, polimorfismo genético, metilentetrahidrofolato reductasa, ácido fólico, homocisteína.

\section{Title}

Study of MTHFR C677T polymorphism in neonates with isolated congenital heart disease in a Colombian population

\begin{abstract}
The research of the role of gene polymorphisms in the metabolic pathways of homocysteine-methionine and folic acid in congenital malformations is very important because its effect could be modulated.
\end{abstract}

Objetive: The aim of this study was to determine whether the C677T polymorphism in the gene of the enzyme methylenetetrahydrofolate reductase (MTHFR) was associated with the development of isolated congenital heart disease.

Methodology: We compared the allele and genotype frequencies of this polymorphism in 34 infants with isolated congenital heart defects and 102 healthy individuals. Genotyping was performed by Polymerase Chain Reaction (PCR) and with the technique Restriction Fragment Length Polymorphism (RFLP).

Results: There were no statistically significant differences in allele or genotype frequencies between case and control groups. Although our results show no statistically significant differences between the groups assessed there was a statistical trend for a possible protective effect of TT genotype against the development of congenital heart disease.

Key words: congenital heart defects, genetic polymorphism, methylenetetrahydrofolate reductasa, folic acid, homocysteine.

\section{Introducción}

La enfermedad cardíaca pediátrica incluye un conjunto de anomalías estructurales y funcionales, que abarca desde malformaciones cardiovasculares o defectos cardíacos congénitos hasta cardiomiopatía, displasia tisular y alteraciones del ritmo cardiaco[1]. Se considera que la enfermedad cardiaca congénita estructural o funcional, se presenta en $1 \%$ de los recién nacidos, pero también está presente en un porcentaje mucho mayor en abortos espontáneos u óbitos fetales[2-4]. Además de su elevada frecuencia, este grupo de malformaciones tiene un impacto importante en la morbilidad y mortalidad pediátricas[5]. La etiología de los defectos cardiacos congénitos es parcialmente comprendida y en gran parte desconocida; los estudios de población sugieren un origen multifactorial al relacionar un sinnúmero de factores con el aumento del riesgo de presentar defectos cardia$\cos [2,4,6]$. Las cardiopatías congénitas pueden asociarse a exposición a teratógenos y síndromes genéticos; sin embargo, la gran mayoría (aproximadamente $80 \%$ ) son catalogadas como no pertenecientes a un síndrome y se cree que su etiología es multifactorial e involucra factores genéticos, epigenéticos y medioambientales $[5,7,8]$.

En la literatura científica se encuentra demostración epidemiológica y experimental que sugiere una posible relación 
entre la alteración del metabolismo de la homocisteína-metionina, con malformación cardiovascular congénita y defectos del tubo neural, estableciendo no solo asociación sino un posible vínculo causal $[4,9]$. Otros estudios han mostrado una asociación estadísticamente significativa entre la presencia del alelo $\mathrm{T}$ del polimorfismo C677T en el gen $M T H F R$, tanto en las madres como en sus recién nacidos. Junker et al. reportaron asociación del genotipo TT de los pacientes con defectos congénitos con estenosis de la válvula pulmonar, síndrome de corazón hipoplásico izquierdo, coartación aórtica y estenosis de válvula aórtica/estenosis subaórtica. van Beynum et al. demostraron asociación entre los genotipos maternos portadores del alelo $\mathrm{T}$ y descendencia con defectos cardiacos cono-truncales, especialmente, cuando la madre no había recibido suplemento de ácido fólico. Zhu et al. reportaron asociación entre el genotipo TT del individuo afectado y aumento de riesgo de conducto arterioso persistente y defecto del tabique interauricular, así como también aumento del riesgo con diferencias estadísticamente significativas de tener hijos con conducto arterioso persistente cuando el genotipo materno era $\mathrm{TT}[2,3,10]$.

El gen MTHFR codifica para la enzima metilentetrahidrofolato reductasa, que cataliza la conversión de 5, 10-metilentetrahidrofolato a 5-metiltetrahidrofolato, precursor crítico en la cascada de reacciones que conducen a la metilación de diversas moléculas, incluyendo el ADN. Además, esta enzima es sumamente importante en la regulación del metabolismo del ácido fólico. Dos polimorfismos de un solo nucleótido (SNP) en el gen que codifica para MTHFR, C677T y A1298C, se han asociado con disminución en la actividad de este enzima. El polimorfismo MTHFR C677T fue identificado en 1995 por Frosst et al.[11] y se caracteriza por la sustitución de la base citosina (C) por timina (T) en el nucleótido de la posición 677 del gen, lo que resulta en el cambio del aminoácido alanina por el aminoácido valina en el dominio catalítico de la MTHFR. Este cambio produce una proteína con acción enzimática reducida, lo que resulta en niveles más altos de homocisteína plasmática.

Es probable que los individuos que presentan el polimorfismo MTHFR C677T tengan un déficit funcional de folato, aun teniendo niveles de folato normales. En estos individuos, el suplemento nutricional con folato a dosis altas podría ser necesario y útil para aumentar los niveles de folato plasmáticos por encima de lo normal y prevenir, de esta manera, la aparición de defectos congénitos[6].

Finalmente, este estudio se planteó con el objetivo de evaluar la asociación entre la presencia del polimorfismo MTHFR C677T en el paciente y la presentación de cardiopatía congénita. 


\section{Material y métodos}

\section{Población de estudio}

Se estudiaron 34 pacientes recién nacidos (casos) que presentaban malformaciones congénitas cardiacas aisladas, provenientes de la unidad de cardiología de la Clínica Abbot Shaio, durante el periodo de octubre de 2008 a diciembre de 2008, previo consentimiento informado. Se evaluaron 102 individuos sanos (controles) sin antecedentes de enfermedades congénitas cardiacas, en apariencia sanos, de ambos sexos, provenientes de diversos lugares de Colombia y con residencia en la ciudad de Bogotá, y edades comprendidas entre 18 y 50 años, previo consentimiento informado.

Los comités de ética de la Pontificia Universidad Javeriana y de la Fundación Clínica Shaio aprobaron el estudio que se llevó a cabo con los lineamientos de la declaración de Helsinki y la Resolución 8430 de 1990 del Ministerio de la Protección Social de Colombia.

Los criterios de inclusión aplicados fueron: pacientes recién nacidos con cardiopatías congénitas aisladas que requirieran cirugía de corazón abierto y los criterios de exclusión eran aquellos pacientes que tuvieran cardiopatías congénitas explicadas por cromosomopatías, antecedentes de teratógenos, síndromes conocidos o familiares.

\section{Determinación del polimorfismo}

A partir de $5 \mathrm{ml}$ de sangre venosa periférica, se extrajo el ADN mediante la técnica de salting out. Se amplificó por PCR un segmento del gen MTHFR de 198 pares de bases, que contiene la región donde se encuentra el polimorfismo de nucleótido único C677T. Se utilizaron como cebadores las secuencias descritas por Bermúdez et al.[12]: 5'TG AAGGAGAAGGTGTCTGCGGGA3' y 5'AGGACGGTGCGGTGAGAG3', en una mezcla de reacción con 44 ng de ADN, 0,5 pmol de iniciadores, 0,08 $\mathrm{mM}$ de dNTP, 2,5 U de Taq polimerasa (Promega) y agua para un volumen final de 25 ul. Luego de una desnaturalización inicial del $\mathrm{ADN}$ a $95^{\circ} \mathrm{C}$ durante cuatro minutos, se realizaron 35 ciclos de desnaturalización a $95^{\circ} \mathrm{C}$ por 30 segundos, anillamiento a $62^{\circ} \mathrm{C}$ por un minuto y extensión a $72^{\circ} \mathrm{C}$ por 30 segundos, en un termociclador iCycler, marca BioRad. Los productos de amplificación se sometieron a electroforesis en geles de agarosa al 1,5\% y se visualizaron mediante tinción con bromuro de etidio e iluminación ultravioleta. Luego se digirió el fragmento con la enzima de restricción TaqI (Promega), utilizando $5 \mathrm{U}$ de enzima y 3 ul de producto de PCR, para un volumen final de $20 \mathrm{ul}$, y se incubó a $65^{\circ} \mathrm{C}$ por cuatro horas. Los fragmentos se sometieron a electroforesis en geles de poliacrilamida al $12 \%$, se tiñeron en bromuro de etidio y se visua- 
lizaron en lámpara de luz ultravioleta. La presencia del polimorfismo crea un sitio de restricción y genera fragmentos de 173 y 25 pares de bases (figura 1).

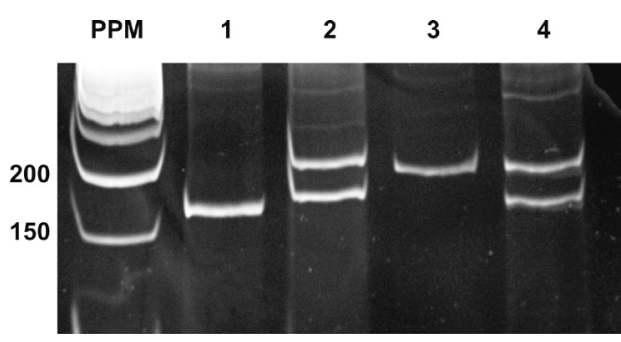

Figura 1. Genotipificación del polimorfismo MTHFR C677T.

Figura 1. Electroforesis en gel de poliacrilamida al $12 \%$ del fragmento amplificado, después de corte con la enzima de restricción TaqI. Cuando el genotipo del individuo es: $\mathrm{T} / \mathrm{T}$ se observa una sola banda de $173 \mathrm{pb}$ (carril 1), C/T dos bandas de 173 y 198 pb (carriles 2 y 4) y cuando es C/C se observa una sola banda de 198 pb (carril 3), PPM: Marcador de peso molecular $50 \mathrm{pb}$.

\section{Análisis estadístico}

Para la obtención de las muestras se consideró la facilidad para su recolección y se calculó el tamaño de muestra de acuerdo con el menor riesgo con diferencias estadísticamente significativas de los odds ratio reportados por Junker et al. (OR 6,1; $\mathrm{IC}_{95 \%}$ : 1,4-27,5; $\mathrm{p}=0,034)$ para diferentes anomalías cardiacas congénitas en niños homoci- gotos TT[3]. Se usó la frecuencia del genotipo TT en población colombiana $(27,47 \%$, dato no reportado) encontrada por Bermúdez et al., relación caso/control 1:3 con nivel de confianza de $95 \%$ y potencia de 95\%[12]. Finalmente se hizo análisis con corrección de Yates y se obtuvo un valor de $\mathrm{n}$ con de $35 \mathrm{ca}-$ sos y 105 controles. El cálculo se hizo con el programa EPIDAT versión 3.1. Se calcularon las frecuencias alélicas y fenotípicas por conteo y se compararon entre los grupos de estudio por la prueba de ji al cuadrado, estableciendo un nivel de significancia del $95 \%$ y utilizando el programa EPIDAT versión 3.1. Para evaluar el equilibrio de HardyWeinberg en las frecuencias genotípicas, se utilizó el programa estadístico Genepop versión 3.4.

\section{Resultados}

Las frecuencias alélicas y genotípicas para el polimorfismo MTHFR C677T entre los grupos de casos y controles, fueron las siguientes: en el grupo de $\operatorname{casos}(\mathrm{n}=34$, alelos $=68)$, se encontraron $37(54,4 \%)$ alelos $\mathrm{C}$ y $31(45,5 \%)$ alelos T; en el grupo control $(\mathrm{n}=102$, alelos $=204)$, se encontraron $97(47,5 \%)$ alelos C y 107 (52,4\%) alelos T, sin diferencias estadísticamente significativas entre los grupos (OR 0,759; $\mathrm{IC}_{95 \%}$ : $0,437-1,317 ; p=0,3269)$ (tabla 1). En el grupo de casos, se encontró el genotipo $\mathrm{CC}$ en 7 niños (20,5\%), el genotipo 
CT en $23(67,6 \%)$ y el genotipo TT en $4(11,7 \%)$; en el grupo control, se encontró el genotipo $\mathrm{CC}$ en 23 individuos (22,5\%), el genotipo CT en $51(50 \%)$ y el genotipo TT en $28(27,4 \%)$, sin diferencias estadísticamente significativas entre los grupos $(\mathrm{p}=0,1262)$ (tabla 1$)$. Se hizo una comparación entre los casos y controles de acuerdo a ser homocigoto TT o no serlo, según la condición de ser o no ser homocigoto TT; en cuanto a este análisis, no se encontraron diferencias estadísticamente significativas entre los grupos de casos y controles (OR: 0,$\left.352 ; \mathrm{IC}_{95 \%}: 0,113-1,091 ; \mathrm{p}=0,0665\right)$; sin embargo, considerando que el valor de p para este último análisis es cercano a 0,05 y la observación de Hobbs et al.[7], la cual postula que una disminución en la actividad de la MTHFR fetal puede tener un efecto protector durante la cardiogénesis cuando la proliferación celular es rápida y se requiere que la síntesis de ADN esté libre de errores, podríamos sospechar un efecto protector del genotipo fetal TT, que sería evidente al aumentar el tamaño de muestra. Las frecuencias genotípicas en ambos grupos se encontraron en equilibrio de Hardy-Weinberg.

Tabla 1

Frecuencias genotípicas y alélicas del polimorfismo C677T del gen que codifica para la MTHFR en casos y controles

\begin{tabular}{lrrrrrr}
\hline & \multicolumn{5}{c}{ Frecuencias genotípicas } & \multicolumn{2}{l}{ Frecuencias alélicas } \\
\hline Grupo & $\mathrm{C} / \mathrm{C} \mathrm{N}(\%)$ & $\mathrm{C} / \mathrm{T} \mathrm{N}(\%)$ & $\mathrm{T} / \mathrm{T} \mathrm{N}(\%)$ & $\mathrm{C} \mathrm{N}(\%)$ & $\mathrm{T} \mathrm{N}(\%)$ & Valor de p \\
\hline Casos & $7(20,5)$ & $23(67,6)$ & $4(11,7)$ & $37(54,4)$ & $31(45,5)$ & $\mathrm{p}^{1}=0,3269$ \\
Controles & $23(22,5)$ & $51(50,0)$ & $28(27,4)$ & $97(47,5)$ & $107(52,4)$ & $\mathrm{p}^{2}=0,1262$ \\
\hline
\end{tabular}

$\mathrm{p}^{1}$ : valor de $\mathrm{p}$ al comparar las frecuencias alélicas entre los grupos de casos y controles. $\mathrm{p}^{2}$ : valor de $\mathrm{p}$ al comparar las frecuencias genotípicas entre los grupos de casos y controles.

\section{Discusión}

El estudio de enfermedades donde están involucradas las vías metabólicas de la homocisteína-metionina y el ácido fólico, asociado a polimorfismos genéticos de las enzimas que participan en estas vías, pueden ser moduladas con intervenciones terapéuticas y nutricionales. La prevalencia del polimorfismo 
MTHFR C677T varía dependiendo de la población estudiada. Mientras en la población afroamericana se han reportado frecuencias para el alelo $\mathrm{T} \mathrm{y}$ genotipo TT de 0,11 y $0,00 \%$ respectivamente, se ha encontrado una mayor frecuencia alélica en la población italiana (44 a 47\%); en Estados Unidos este es más frecuente en la población hispana que prevalece en Atlanta y California (41,1 y 42\%), así como en la población francesa y japonesa (36 y $34 \%$, respectivamente) $[5,6,10]$. En la población mexicana, el mismo alelo es más frecuente que en otras poblaciones; en el grupo étnico mestizo se presenta entre 50 y $58,5 \%$ y se ha reportado una frecuencia genotípica para el homocigoto TT de 32 a 35,7\% en México[5]. En Colombia, Bermúdez et al., en un grupo de individuos sanos, encontraron que la frecuencia alélica del alelo $\mathrm{T}$ para MTHFR fue de 52,45\% y la frecuencia genotípica de TT fue de 27,45\% (datos no publicados)[12].

Respecto a las limitaciones de este estudio, es posible que se deba aumentar el tamaño de la muestra, considerando las recomendaciones de A. C. Pereira et al. (2005) en cuanto a la corrección del efecto de la estructura genética de la población, y establecer una población de estudio con un grupo homogéneo de diagnósticos anatómicos[4, 13$]$.

Es importante resaltar la necesidad de adelantar estudios sobre enferme- dades donde se ha encontrado un papel importante del polimorfismo MTHFR C677T, en poblaciones con frecuencias elevadas del alelo T y genotipo TT, como en la población colombiana. La presencia del polimorfismo MTHFR C677T en el genotipo materno, se ha reportado como un factor de riesgo para tener hijos con cardiopatías congénitas, por lo que nuestros resultados, junto con lo reportado en la literatura científica, nos orientan hacia estudios que evalúen el papel de la interacción de los genotipos maternos y fetales en el riesgo de tener un hijo con cardiopatía congénita[14]. Igualmente, los estudios futuros deberán analizar este polimorfismo con otros que estén presentes en genes que codifican para otras moléculas que también participen en las vías metabólicas del folato y la homocisteína-metionina. También, es necesario hacer estudios de interrelación entre variantes genéticas y factores modificadores medioambientales que afecten la síntesis de ADN y su metilación, así como estudios sobre la regulación del efecto del polimorfismo MTHFR C677T en la fisiopatología de la enfermedad, mediante intervenciones terapéuticas y nutricionales, particularmente en las mujeres en edad fértil, a quienes se les debe mostrar la importancia de tener una dieta balanceada que les aporte por lo menos $400 \mu \mathrm{g} / \mathrm{día}$ de ácido fólico y, en caso de déficit se pueda suministrar como suplemento alimenticio. Cabe resaltar la recomendación dada por autoridades en salud 
pública y organizaciones de profesionales de la salud, con respecto a acciones relacionadas con estas vías metabólicas, que contribuyen a reducir el riesgo de embarazos con fetos afectados por defectos del tubo neural, y es probable que en un futuro se logre demostrar la eficacia de medidas similares en la reducción del riesgo de presentar defectos cardiacos congénitos[5,9].

Estos hallazgos deben incentivar para continuar los estudios que aporten mayor conocimiento y mejor comprensión del papel de las vías metabólicas de la homocisteína-metionina y el folato, tanto en el desarrollo de cardiopatías como de otras malformaciones congénitas.

\section{Agradecimientos}

Estela Acevedo, Secretaria, Depto Cardiovascular, Fundación Clínica Shaio

\section{Conflicto de intereses}

Los autores declaran que no hay conflicto de intereses en el presente manuscrito.

\section{Bibliografía}

1. Lin AE, Ardinger HH. Genetic epidemiology of cardiovascular malformations. Prog Pediatr Cardiol. 2005;20:113-26.
2. van Beynum I M, Kapusta L, Den Hei jer M, Vermeulen S.H.H.M, Kouwenberg M, Daniëls O, et al. Maternal MTHFR $677 \mathrm{C}>\mathrm{T}$ is a risk factor for congenital heart defects: Effect modification by periconceptional folate supplementation. Eur Heart J. 2006;27:981-7.

3. Junker R, Kotthoff $\mathrm{S}$, Vielhaber $\mathrm{H}$, Halimeh S, Kosch A, Koch HG, et al. Infant methylenetetrahydrofolate reductase 677TT genotype is a risk factor for congenital heart disease. Cardiovasc Res. 2001;51:251-4.

4. Sánchez-Urbina R, Galaviz-Hernández C, Sierra-Ramírez A, Morán-Barroso VF, García-Cavazos R. Trascendencia de los factores ambientales y genéticos en cardiopatías congénitas: el caso de la enzima MTHFR. Perinatol Reprod Hum. 2006;20:39-47.

5. Lupo P, Goldmuntz F, Mitchell L. Genegene interactions in the folate metabolic pathway and the risk of conotruncal heart defects. J Biomed Biotechnol. 2010; artículo ID 630940. doi:10.1155/2010/630940.

6. Huhta JC, Hernández-Robles JA. Homocysteine, folate, and congenital heart defects. Fetal Pediatr Pathol. 2005;24:71-9.

7. Hobbs CA, James SJ, ParsianA, Krakowian PA, Jernigan S, Greenhaw JJ, et al. Congenital heart defects and genetic variants in the methylenetetrahydroflate reductase gene. J Med Genet. 2006;43:162-6.

8. Hobbs CA, James SJ, Jernigan S, Melnyk S, Yunxia L, Malik S, et al. Congenital heart defects, maternal homocysteine, smoking, and the $677 \mathrm{C}>\mathrm{T}$ polymorphism in the methylenetetrahydrofolate reductase gene: Evaluating gene-environment interactions. Am J Obstet Gynecol. 2006;194:218-24. 
9. Botto LD, Mulinare J, Erickson JD. Do multivitamin or folic acid supplements reduce the risk for congenital heart defects? Evidence and gaps. Am J Med Genet. 2003;121A:95-101.

10. Zhu W, Li Y, Yan L, Dao J, Li S. Maternal and offspring MTHFR gene C677T polymorphism as predictors of congenital atrial septal defect and patent ductus arteriosus. Mol Hum Reprod. 2006;12:51-4.

11. Frosst P, Blom HJ, Milos 1 . A candidate genetic risk factor for vascular disease: A common mutation in methylenetetrahydrofolate reductase. Nat Genet. 1995;10:111-3.

12. Bermúdez M, Briceño I, Gil F, Bernal J. Homocisteína y polimorfismos de cista- tionina $\mathrm{b}$ sintasa y metilentetrahidrofolato reductasa en población sana de Colombia. Colomb Med. 2006;37:46-52.

13. Pereira AC, Xavier-Neto J, Mesquita SM, Mota GF, Lopes AA, Krieger JE. Lack of evidence of association between MTHFR C677T polymorphism and congenital heart disease in a TDT study design. Int $J$ Cardiol. 2005;105:15-8.

14. García-Fragoso L, García-García I, Leavitt G, Renta J, Ayala MA, Cadilla LC. MTHFR polymorphisms in Puerto Rican children with isolated congenital heart disease and their mothers. International Journal of Genetics and Molecular Biology. 2010;2:43-7. 